\title{
EVALUATION OF MANET PERFormanCE IN Presence Of Obstacles
}

\author{
Chirag Kumar ${ }^{1}$, Bharat Bhushan ${ }^{2}$, Shailender Gupta ${ }^{3}$ \\ ${ }^{1}$ Department of Electronics Engineering, YMCAUST, Faridabad, India \\ chiragarora35egmail.com \\ ${ }^{2}$ Department of Electronics Engineering, YMCAUST, Faridabad, India \\ bhrts@yahoo. com \\ ${ }^{3}$ Department of Electronics Engineering, YMCAUST, Faridabad, India \\ shailender81@gmail.com
}

\begin{abstract}
The mobility models are used to represent the unpredictable movement pattern of the nodes in Mobile Adhoc Network (MANET) and give us an idea regarding their location, velocity and acceleration change over time. These models are used for simulation purpose in standard software tools such as QualNet, ns-2 etc. This paper evaluates the performance of routing protocols for mobility models such as Random Way Point (RWP), Random Walk (RW) and Random Direction (RD) in presence of obstacles like mountain which restricts node movement as well obstruct transmission path between nodes based on a parameter termed as Probability of Reachability (POR). The POR is defined as the fraction of reachable routes to all possible routes between all pairs of sources and destinations. For this purpose a simulator is designed in MATLAB. We observe a marked difference in value of POR in presence of obstacles as well as variation in number of obstacles.
\end{abstract}

\section{KEYWORDS}

MANET, Obstacle, Mobility Model, POR

\section{INTRODUCTION}

MANET [1] is formed by the set of mobile nodes that are connected via wireless links without using any fixed infrastructure. Due to absence of centralized routers, each node in MANET has to act as a gateway, transmitter and receiver, making the routing task even more challenging than other conventional wireless networks. In addition to above several other factors such as areas shape where the network is to be deployed [2], limited bandwidth, processing capability, memory, battery power, and unpredictable movement of the nodes also affect the reachability significantly. The unpredictable behaviour of nodes in MANET results in their random organization which alters the topology of the network rapidly and unpredictably. This unpredictable movement pattern of nodes is presented by various researchers by presenting an idea regarding their location, velocity and acceleration change over time and is termed as mobility model [3] [4].

To evaluate the performance of mobility models on routing protocols, network simulator are designed which makes simulation modelling an invaluable tool for understanding the operation of these networks. Once the nodes in these networks are placed, the mobility model determines how the nodes move within the network. A variety of mobility models have been proposed for MANET [5, 6, 7, 8, 9, 10], and a survey of many is presented in $[3,4,11]$. These models vary widely in their movement characteristics. All these models play a significant role in determining the reachability of routing protocols. 
International Journal of Ad hoc, Sensor \& Ubiquitous Computing (IJASUC) Vol.3, No.3, June 2012

Literature study $[4,11,12,13,20]$ has shown that the mobility model in use can significantly impact the performance of ad hoc routing protocols, based on packet delivery ratio, the control overhead, and the data packet delay. Hence, it is important to use mobility models that accurately represent the intended scenarios in which the protocol is likely to be used. In this way the performance of the protocol can be more accurately predicted. In this paper, we propose to create more realistic movement models by incorporating obstacles in the simulation area. The obstacles $[14,20]$ are placed within a network area to model the location of buildings within an environment, i.e. a college campus. This paper studies the impact of mobility models such as Random Way Point (RWP), Random Walk (RW) and Random Direction (RD) under the presence of obstacles on reachability of routing protocols.

The paper has been organized as follows: Section 2 provides the literature survey on mobility models used in our simulation, section 3 provides the simulator design and experimental setup parameters and section 4 describes the simulation and results followed by concluding remarks.

\section{MobILITY MODELS USED IN MANET}

The various mobility models used to evaluate the performance (POR) of routing protocols are as follows

\subsection{Random Way Point Mobility Model (RWP)}

The RWP model was proposed by John and Maltz[15] in which all the nodes randomly select different locations as their destinations within the simulation area [3][4][16]. With the start of simulation the nodes start moving towards the selected destinations from their existing locations with uniform velocities selected randomly from the uniformly distributed array $\left[0, \mathrm{~V}_{\max }\right]$. Once the node reaches at the destination, it stays there for some time known as pause time before moving to a new destination. The pause time is selected from the array $\left[0, \mathrm{~T}_{\text {pause }}\right]$. The above process is repeated until the simulation time is over. In RWP model the behaviour of the mobile nodes is completely described by the maximum velocity $\left(\mathrm{V}_{\max }\right)$ and the Pause Time $\left(\mathrm{T}_{\text {pause }}\right)$. Fig 1 shows the movement of a node using RWP Mobility Model.

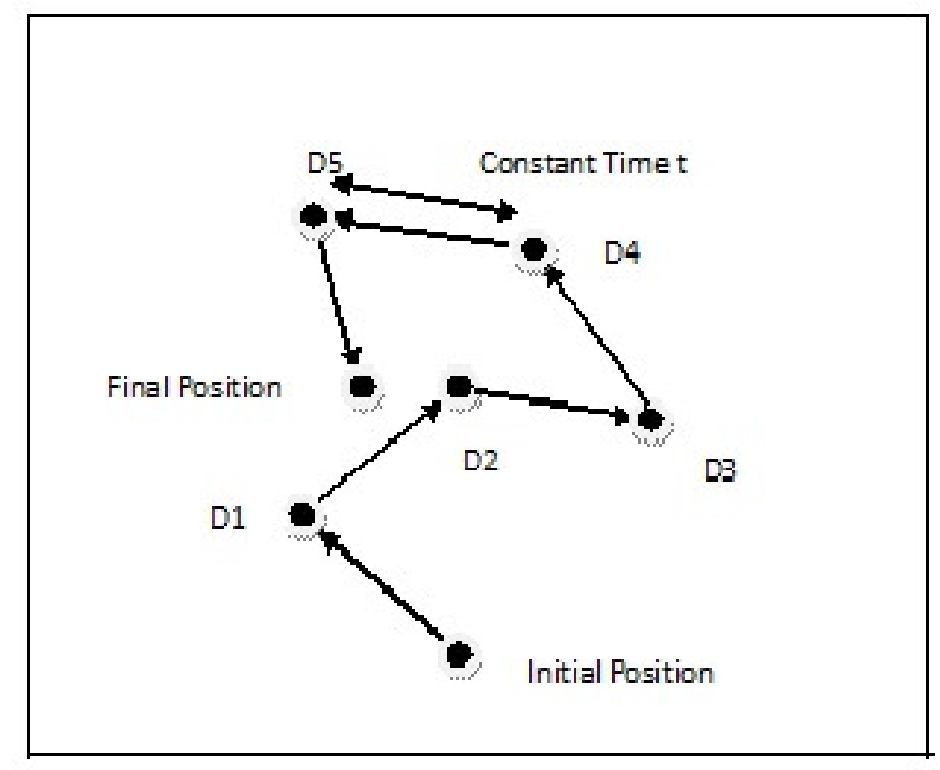

Figure 1. Node movement in Random Way Point Mobility Model 
International Journal of Ad hoc, Sensor \& Ubiquitous Computing (IJASUC) Vol.3, No.3, June 2012

\subsection{Random Walk Mobility Model (RW)}

This mobility model [4][16][17] was developed and described mathematically by Einstein in 1926 to emulate the unpredictable movements of the particles known as Brownian motion . In this model a node starts its motion by selecting a direction with speed from the pre-specified ranges $\left[0,2^{*} \pi\right]$ and $\left[0, \mathrm{~V}_{\max }\right]$. The node moves for a fixed time interval $\mathrm{t}$ or moves for a fixed distance $\mathrm{d}$. After distance $\mathrm{d}$ or time $\mathrm{t}$, new direction and speed are selected from the prespecified ranges. If the specified time or distance is very small then the node's movement pattern will be restricted to a small portion of the simulation area and vice versa. If a particular node reaches to the boundary of simulation area it is bounced back with $\pi$-incoming angle and is termed as border effect. This model is a memory-less. Therefore, the current speed and the direction of the node is independent of its past speed and direction. Fig 2 shows the movement of a node in case of RW Mobility Model.

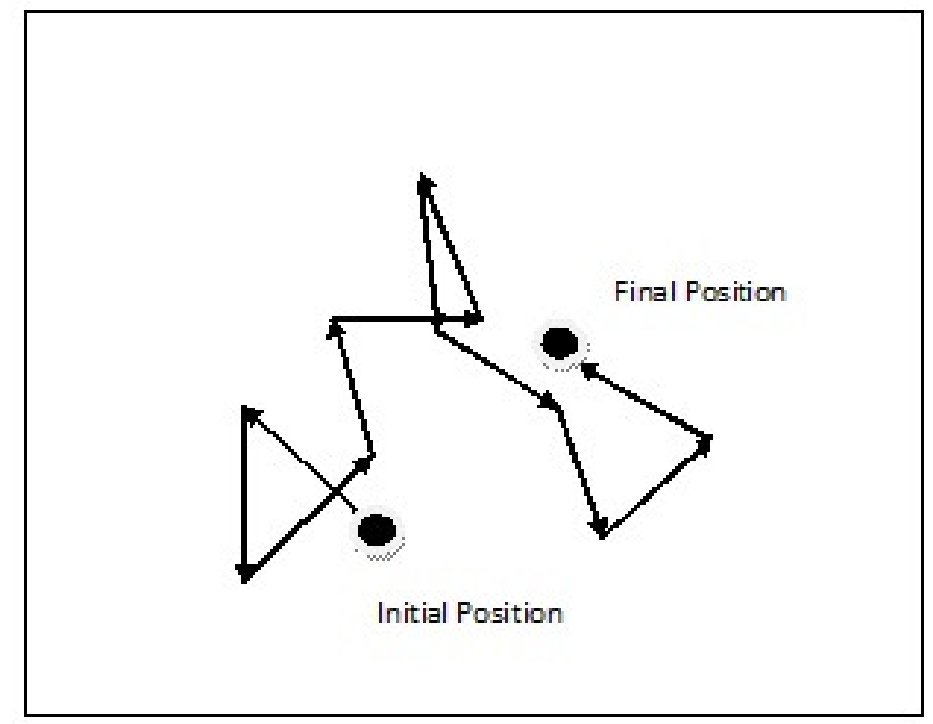

Figure 2. Node movement in Random Walk Mobility Model

\subsection{Random Direction Mobility Model (RDM)}

The random direction mobility model [3][16][18] was developed in order to over-come the flaws discovered in the RWM Model. The problem in the RWM Model is that the probability of a node to choose a new destination located at the centre of the simulation area, or a destination that requires path of the node through the centre of the simulation area is high. This results in clustering of mobile nodes near the centre of simulation area. In RDM model the node chooses random direction and velocity from the specified range $[0,2 * \pi]$ and $\left[0, \mathrm{~V}_{\max }\right]$. As the node reaches at the border of the simulation area, it waits there for pause time. After expiry of pause time, node chooses a new direction from $[0, \pi]$ and starts moving again to-wards the simulation border in a new direction. This process continues until the simulation is over. This mobility model is similar to the RWM Model, with a small difference in motion of a node to the border of simulation in RD model instead of motion for constant time or distance as in RWM. Fig 3 shows the movement of a node in case of RD Mobility Model. 


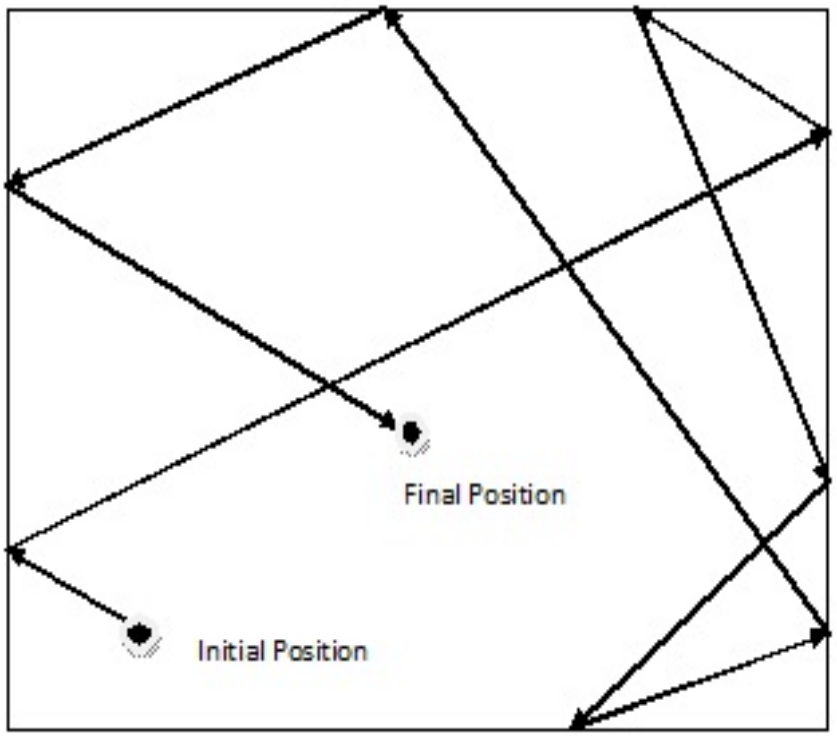

Figure 3. Node movement in Random Direction Mobility Model

\section{Simulation AND EXPERIMENTAL SETUP}

\subsection{Metric Used}

The Probability of Reachability (POR) [19] is used to evaluate the performance of different mobility models in idealistic and realistic conditions and is defined as "The fraction of possible reachable routes to all possible routes between all different sources to all different destinations". Mathematically it can be expressed as

$$
\text { POR }=\frac{\text { EREACHAELE FATHS EETWEEN SOURCE AHU DESTINATION }}{\text { (1) }}
$$

where ' $n$ ' is number of nodes.

The POR is calculated by checking the path existence between all pairs of sources and destinations. For this purpose a count variable is taken and initialized to zero value. If the path exists, variable count is incremented. In this way all combinations of source destination pairs are checked. The POR is calculated by using the above equation. To ensure complete randomness the process is repeated 25 times and average POR is calculated as shown in Fig. 4.

\subsection{Simulation Setup Parameter}

Various Parameters used for the simulation process are as given in Table 1.

\subsection{Simulation Process}

In Fig 4 the simulation process is explained using flowchart. Fig 5 and Fig. 6 shows the snapshot of the simulation area, where MANET nodes are to be deployed in and absence of obstacles using MATLAB as simulation tool. The path in red colour shows the Minimum hop routing between the nodes. The green colour rectangle represents the obstacles in the simulation region. 
International Journal of Ad hoc, Sensor \& Ubiquitous Computing (IJASUC) Vol.3, No.3, June 2012

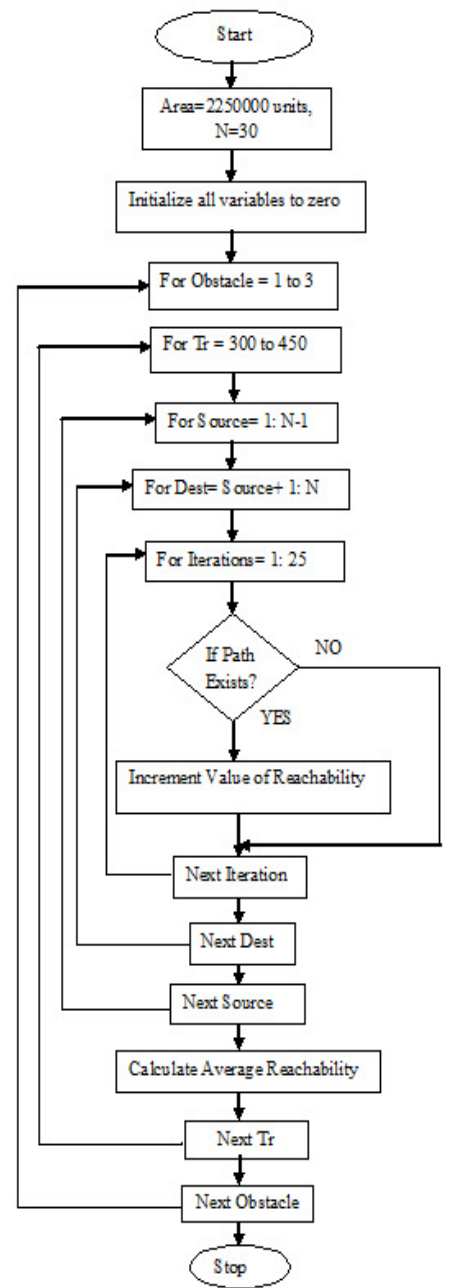

Figure 3. Flowchart

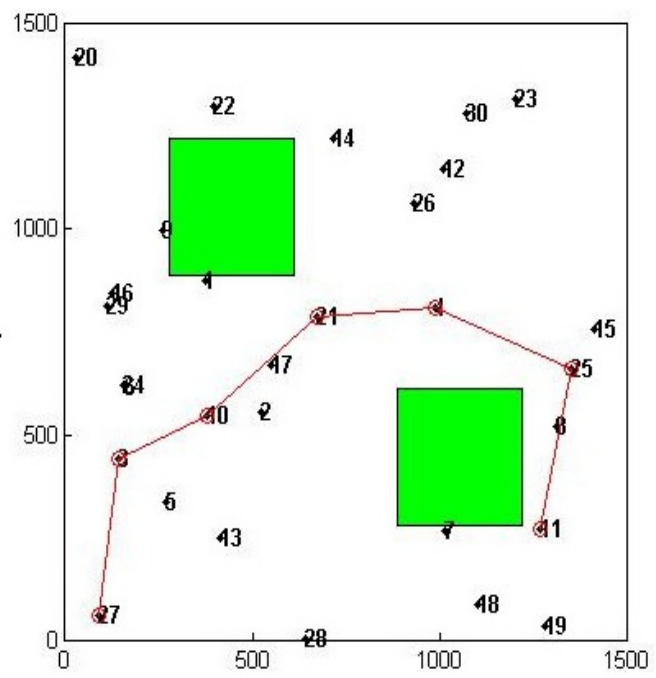

Figure 6(b).

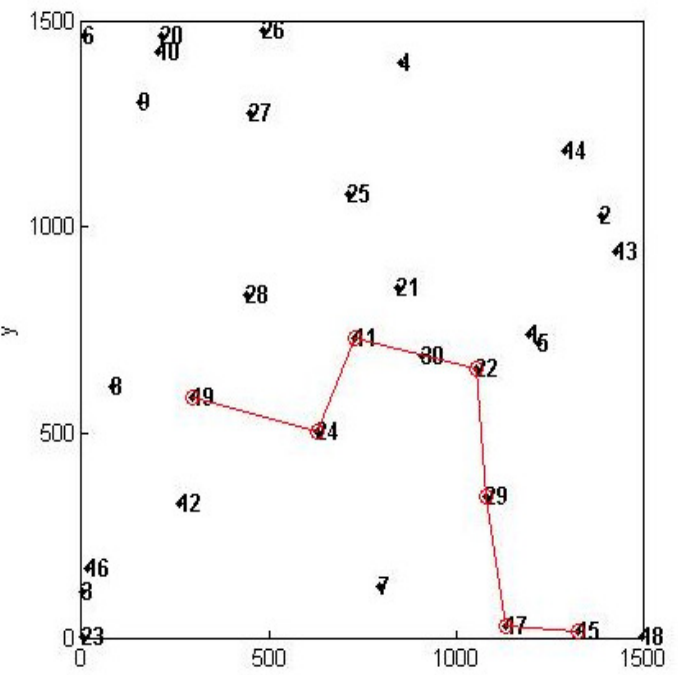

Figure 5. Snapshot for Absence of Obstacle
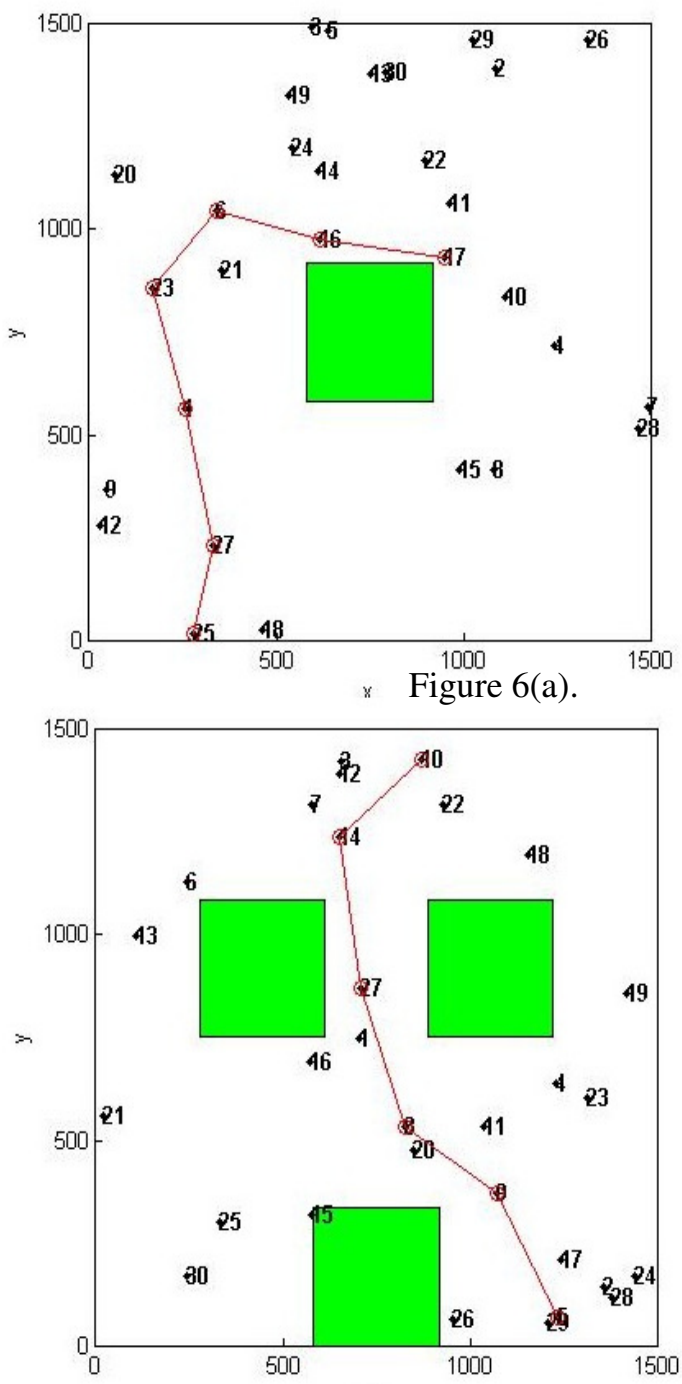

Figure 6. Snapshot for Variation in Number ${ }^{x}$ Figure 6(c).

Obstacles 
Table 1. Simulation setup Parameters.

\begin{tabular}{|l|l|}
\hline Parameter & Value \\
\hline Size of Region & 2250000 sq. unit \\
\hline Mobility Model Used & RWP, RW, RD. \\
\hline Number of Nodes Deployed & 30 \\
\hline Number of Obstacles & 1 to 3 \\
\hline Obstacle Type & $\begin{array}{l}\text { Obstructing Transmission Range } \\
\text { Type }\end{array}$ \\
\hline Obstacle Area & $\begin{array}{l}\text { Square Shaped Area = 335.41 X } \\
335.41\end{array}$ \\
\hline Transmission Range & 300 to 450 step size 50 \\
\hline Routing Algorithm Used & $\begin{array}{l}\text { Dijkstra's Shortest Path Routing } \\
\text { Algorithm }\end{array}$ \\
\hline Routing Strategies Used & Minimum Hop Routing \\
\hline Placement of Nodes & Random \\
\hline Number of iterations & 25 \\
\hline
\end{tabular}

\subsection{Results}

\subsubsection{Impact of one obstacle on Mobility Model}

Fig. 7 shows the impact of varying transmission range on Minimum Hop routing protocol in the presence of single obstacle. It can be easily observed from the graph that as the transmission range increases the value of POR increases. This is due to the fact with increase in transmission range of the nodes the neighbour node density increases, resulting in increase in the value of reachability. Another interesting point to be noted here is that in presence of single obstacle there is only a marginal difference in the performance of mobility models.

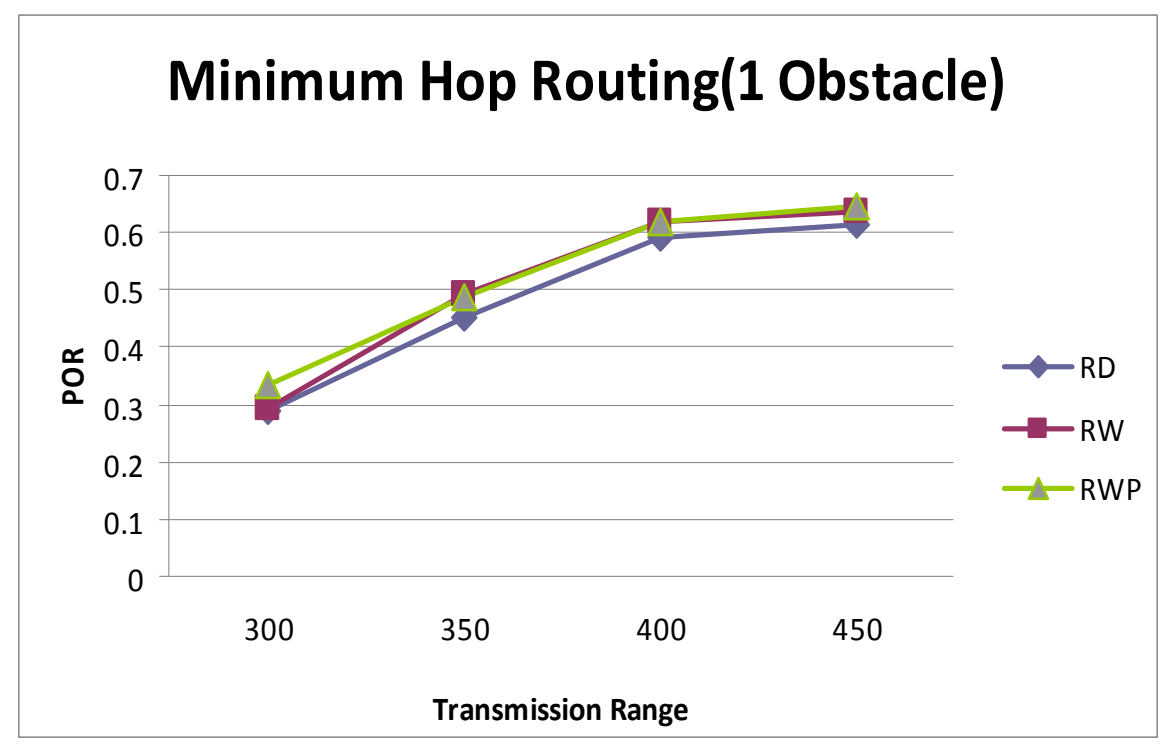

Figure 7. Value of POR for mobility models in presence of single obstacle. 
International Journal of Ad hoc, Sensor \& Ubiquitous Computing (IJASUC) Vol.3, No.3, June 2012

\subsubsection{Impact of two obstacles on mobility model}

Fig. 8 shows the impact of varying transmission range on the value of POR by placing two obstacles in the simulation region using Minimum Hop routing protocol. It can be observed from the graph that as the transmission range increases, the value of POR increases. Also from the comparison of three mobility models, it is observed that RWP mobility model has best POR performance followed by RD \& RW mobility models.

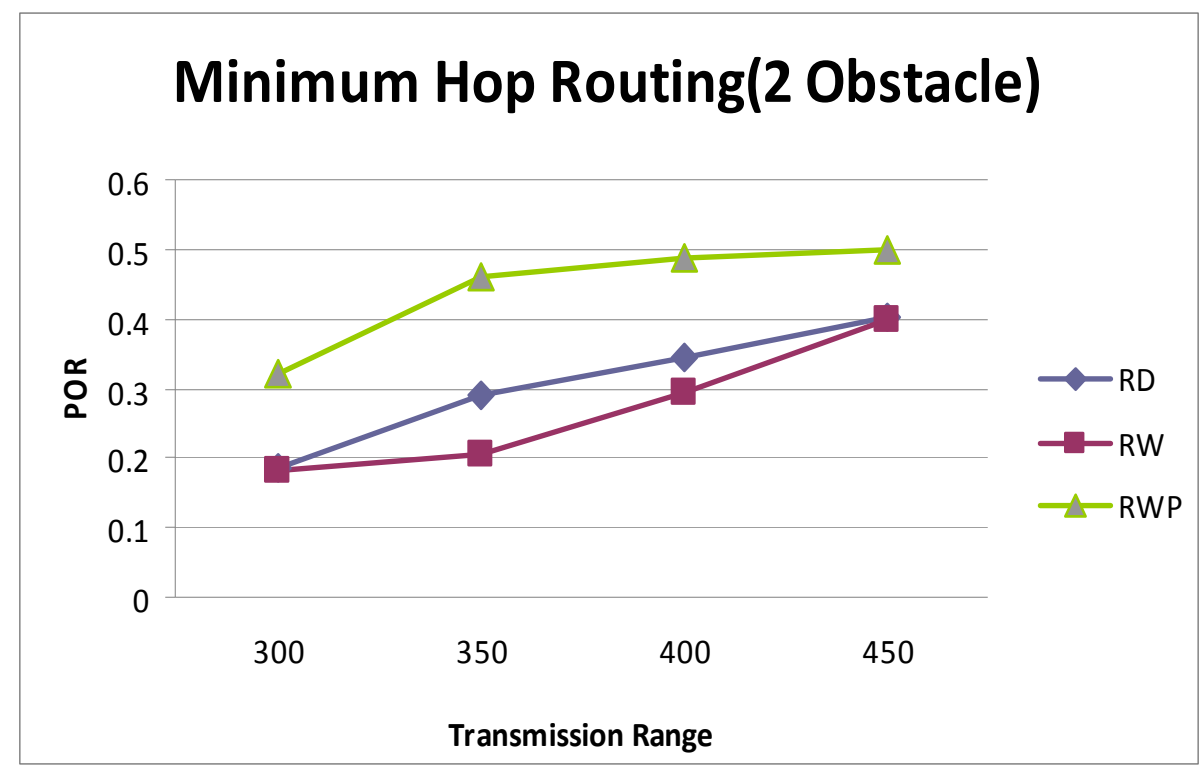

Figure 8. Value of POR for mobility models in presence of two obstacles

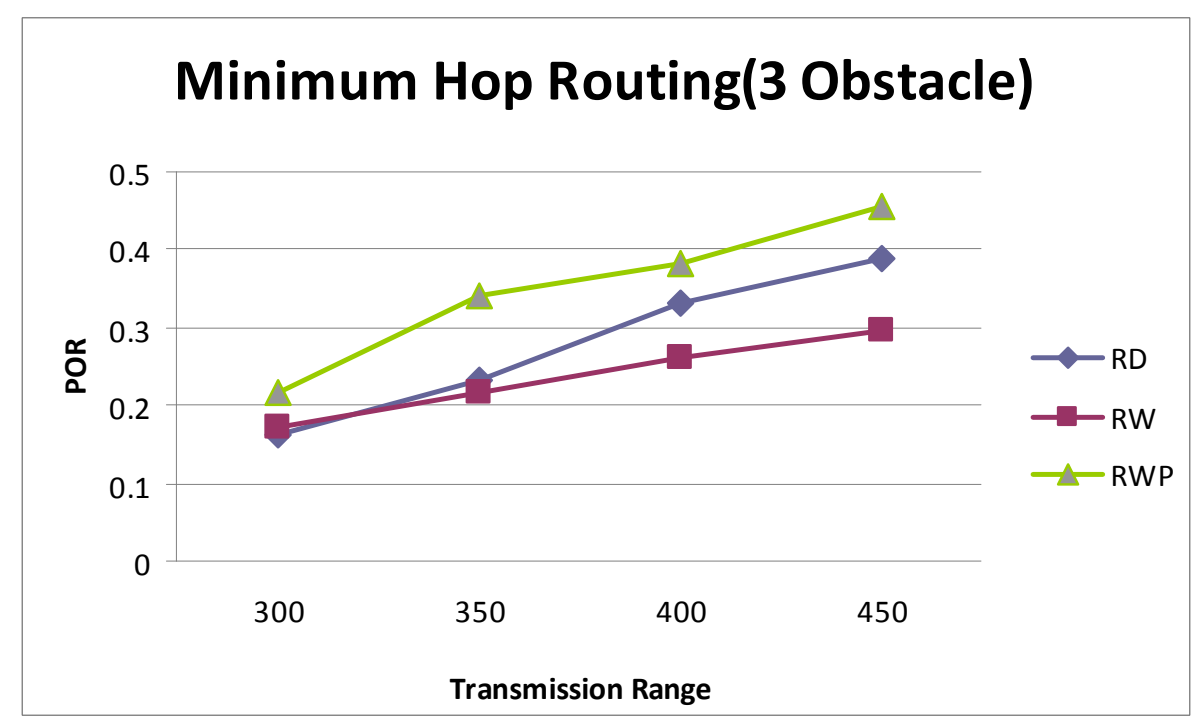

Figure 9. Value of POR for mobility models in presence of three obstacles

\subsubsection{Impact of three obstacles on mobility model}

Fig. 9 shows the impact of varying transmission range on the value of POR for three mobility models using Minimum hop routing protocol by employing three obstacles in simulation region. 
International Journal of Ad hoc, Sensor \& Ubiquitous Computing (IJASUC) Vol.3, No.3, June 2012

Once again it is observed that with increase of transmission range the value of POR increases for all mobility models and RWP models performs best in terms of POR performance followed by RD \& RW mobility models.

\subsubsection{Impact of number of obstacles on POR}

Fig 10 shows the values of POR for all three mobility models in presence of varying number of obstacles for given transmission range (450 units). It can be observed that as the number of obstacles increases, more number of obstructions are created between nodes leading to decrease in the POR value. On comparison of three mobility models the RWP model performs best on the basis of POR value.

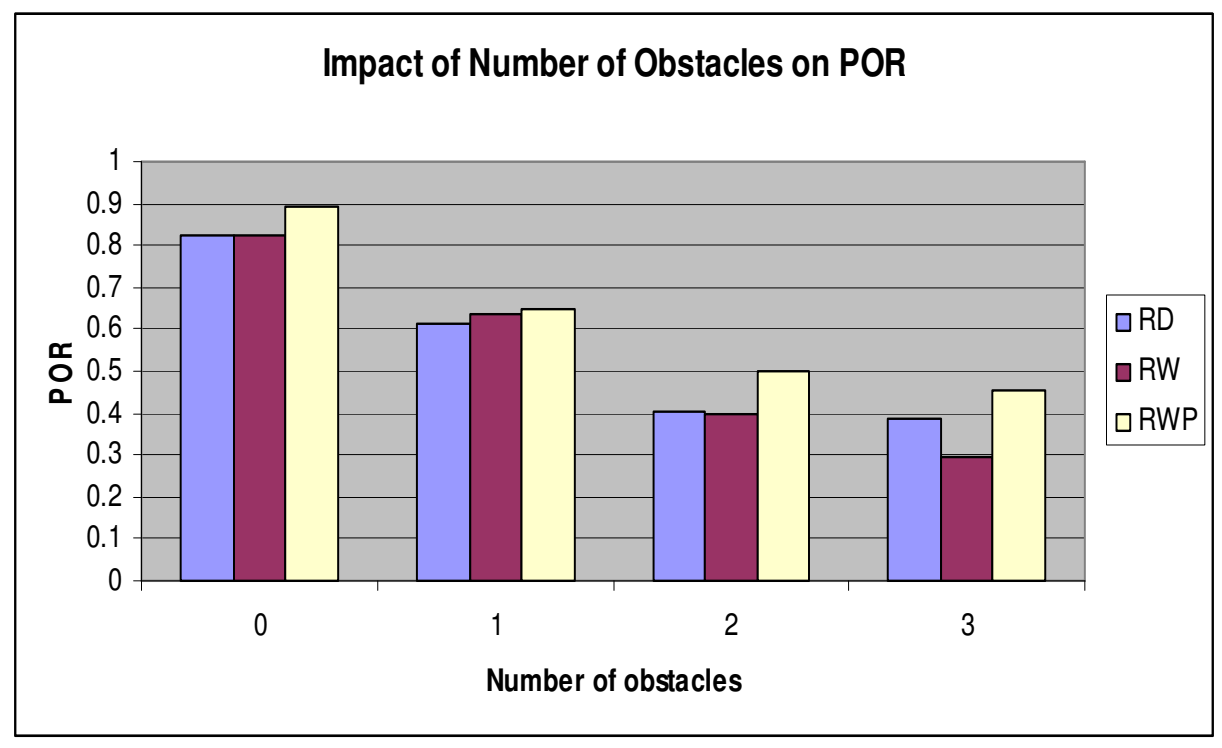

Figure 10. POR vs. number of obstacles

\section{Conclusions}

From the various simulation results the concluding remarks can be made as follows:

- The RWP mobility model is having highest POR in absence as well as in presence of one, two and three number of obstacles.

- At lower value of transmission range the POR value of all the three models is significantly low. This is due to the fact that at lower transmission range the number of neighbour per node is quite low.

- The presence of obstacles significantly reduces the POR value, since the obstacles not only restricts the node movement but at the same time reduces the effective transmission range of the nodes.

It can be concluded that the performance of all the mobility models is very much influenced in the presence of obstacles as can be seen from all the above mentioned results. 
International Journal of Ad hoc, Sensor \& Ubiquitous Computing (IJASUC) Vol.3, No.3, June 2012

\section{REFERENCES}

[1] Joseph Macker and Scott Corson,"Mobile ad-hoc networks (MANET)", http://www.ietf.org/proceedings/01dec/183.htm, December 2001.

[2] C. K. Nagpal, Shailender Gupta, and Bharat Bhushan,'Impact of Area's Shape on MANET Performance",IEEE Conference WICT 2011.

[3] Tracy Camp, Jeff Boleng, Vanessa Davies,” A Survey of Mobility Models for Ad Hoc Net-work Research", Wireless Communication \& Mobile Computing (WCMC): Special issue on Mobile Ad Hoc Networking:Research, Trends and Applications, vol. 2, no. 5, pp. 483-502, 2002.

[4] Nicholas Cooper and Natarajan Meghanathan,"Impact of Mobility Models in Multi-Path Routing in Mobile Ad Hoc Networks",International Journal Of Computer Networks \& Communications (IJCNC), Vol. 2, No.1, January 2010.

[5] J. Broch, D. A. Maltz, D. Johnson, Y.-C. Hu, and J. Jetcheva," A Performance Comparison of Multi-Hop Wireless Ad Hoc Network Routing Protocols",In Proceedings of the 4th Annual ACM/IEEE International Conference on Mobile Computing and Networking (MobiCom), pages 85-97, Dallas, Texas, October 1998.

[6] V. Davies,"Evaluating Mobility Models Within an Adhoc Network. Master's thesis, Colorado School of Mines", 2000.

[7] Z. Haas,"A New Routing Protocol for Reconfigurable Wireless Networks",In Proceedings of the IEEE International Conference on Universal Personal Communications (ICUPC), pages 562565, October 1997.

[8] X. Hong, M. Gerla, G. Pei, and C.-C. Chiang,"A Group Mobility Model for Ad hoc Wireless Networks", In Proceedings of the ACM/IEEE MSWIM'99, Seattle, WA, August 1999.

[9] B. Liang and Z. Haas,"Predictive Distance-based Mobility Management for PCS Networks", In Proceedings of the IEEE Conference on Computer Communication (INFOCOM), New York, NY, March 1999.

[10] E. M. Royer, P. M. Melliar-Smith, and L. E. Moser,"An Analysis of the Optimum Node Density for Ad hoc Mobile Networks", In Proceedings of the IEEE International Conference on Communications, pages 857-861, Helsinki, Finland, June 2001.

[11] Santosh Kumar, S C Sharma Bhupendra Suman,"Classification and Evaluation of Mobility Metrics for Mobility Model Movement Patterns in Mobile Ad-Hoc Networks",International journal on applications of graph theory in wireless ad hoc and sensor networks (GRAPH-HOC) Vol.3, No.3, September 2011.

[12] Bhavyesh Divecha, Ajith Abraham, Crina Grosan and Sugata Sanyal,"Impact of node mo-bility on MANET routing protocols models" ,Journal of Digital Information Management, Feb 12007.

[13] Aravindhan Venkateswaran, Venkatesh Sarangan, Natarajan Gautam and Raj Acharya,"Impact of mobility prediction on the temporal stability of MANET clustering algorithms", Proceedings of the 2nd ACM international workshop on Performance evaluation of wireless ad hoc, sensor, aand ubiquitous networks, 2005.

[14] Amit Jardosh, Elizabeth M. BeldingRoyer,Kevin C. Almeroth, Subhash Suri,"Towards Realistic Mobility Models For Mobile Ad hoc Networks",MobiCom'03, September 14-19, 2003, San Diego, California, USA.).

[15] Random Waypoint Model,http://www.netlab.tkk.fi/ esa/java/rwp/rwpmodel.sht

[16] Mohd Izuan Mohd Saad, Zuriati Ahmad Zukarnain,” Performance Analysis of Random-Based Mobility Models in MANET Routing Protocol", European Journal of Scientific Re-search ISSN 1450-216X Vol.32 No.4 (2009), pp.444-454.

[17] M. Zonoozi and P. Dassanayake. "User Mobility Modeling and Characterization of Mobility Pattern." IEEE Journal on Selected Areas in Communications, 15(7), pages 1239-1252, September 1997. 
International Journal of Ad hoc, Sensor \& Ubiquitous Computing (IJASUC) Vol.3, No.3, June 2012

[18] Shafinaz Buruhanudeen, Mohamed Othman, Mazliza Othman, Borhanuddin Mohd Ali," Mobility Models, Broadcasting Methods and Factors Contributing Towards the Efficiency of the MANET Routing Protocols: Overview",paper id-123.

[19] Chander Kumar, Shailender Gupta and Bharat Bhushan,'IMPACT OF VARIOUS FACTORS ON PROBABILITY OF REACHABILITY IN MANET: A SURVEY”,International journal on applications of graph theory in wireless ad hoc networks and sensor networks (GRAPH-HOC) Vol.3, No.3, September 2011.

[20] Chirag Kumar, C.K. Nagpal, Bharat Bhushan, Shailender Gupta,"Reachability Analysis of Mobility Models under Idealistic and Realistic Environments", published in proceedings by springer, wimoa-2012,page no. 519-528.

\section{Authors}

Mr. Chirag Kumar has B. Tech (ECE) from Kurukshetra University and pursuing his M.Tech (ECE) from YMCA University of Science and Technology. His academic interests include network security and fuzzy logic.

Mr. Bharat Bhushan has B. Tech (Electronics) from PEC and M.Tech (Electronics) from YMCA University of Science and Technology. His academic interests include Mobile Ad-hoc Network, Network Security.

Mr. Shailender Gupta is B. Tech (Electronics) and M. Tech (Computer Engg.) and pursuing his $\mathrm{Ph} . \mathrm{D}$ in the area of ad-hoc mobile network security from YMCA University of Science and Technology. His academic interests include network security, automata theory and fuzzy logic. 